\title{
Ingenol mebutate in the treatment of actinic keratoses: clearance rate and adverse effects ${ }^{*}$
}

\author{
Maria Isabel Ramos Saraiva ${ }^{1,3}$, Larissa Karine Leite Portocarrero ${ }^{1}$, Marcella Amaral Horta Barbosa Vieira ${ }^{1}$, \\ Bethania Cabral Cavalli Swiczar ${ }^{1}$, Andrezza Telles Westin²
}

DOI: http:/ /dx.doi.org/10.1590/abd1806-4841.20186982

\begin{abstract}
BACKGROUND: Actinic keratoses are benign intraepithelial skin neoplasms that develop in photoexposed areas and can progress to invasive carcinoma. They are seen frequently in dermatological practice, occurring in $5.1 \%$ of consultations. Ingenol mebutate (IM) was recently approved in Brazil as a topical therapy for field cancerization in actinic keratosis.

Овјестіvе: To evaluate the clearance rate and adverse events in the treatment of actinic keratoses with ingenol mebutate.

METHODs: A longitudinal, prospective, non-randomized, interventional, open, single-center study was conducted. Patients with actinic keratoses applied ingenol mebutate on a $25 \mathrm{~cm}^{2}$ area of the face and/or scalp for three consecutive days (0.015\%) or on the forearm for two days $(0.05 \%)$.

RESULTS: 27 patients completed the protocol, of whom 13 on the face and/or scalp and 14 on the forearm. Complete clearance occurred in $53.8 \%$ in the first group and $42.8 \%$ in the second. Partial response was observed in 15.4\% and 35.7\%, respectively. The most common side effects were erythema, edema, desquamation, pruritus, and local erosion.

STUDY LIMITATIONS: The study had a small sample and was not randomized, double-blind, placebo-controlled, or vehiclecontrolled.

CONCLUSION: Ingenol mebutate is well-tolerated for the treatment of actinic keratosis, with good patient adherence thanks to the short treatment period.
\end{abstract}

Keywords: Keratosis, actinic; Skin neoplasms; Therapeutics; Treatment outcome

\section{INTRODUCTION}

Skin alterations in response to sun exposure have increased dramatically in the last 50 years due to population aging and increased exposure to ultraviolet (UV) radiation. ${ }^{1}$ Solar keratosis, senile keratosis, or actinic keratosis (AK) are benign intraepithelial neoplasms, formed by atypical keratinocyte proliferation, common in photo-exposed areas. They comprise a significant share of dermatology practice and are considered the fourth most frequent diagnosis, occurring in $5.1 \%$ of patient consultations in Brazilian statistics for $2006 .^{2}$

AK mainly affects individuals with low phototypes and, increasingly, immunosuppressed individuals. ${ }^{2}$ Secondary factors for the development of AK are advanced age, male gender, birthplace with higher UV index, Caucasoid ethnic group, previous history of cutaneous neoplasms, work history with sun exposure, and low socioeconomic status. ${ }^{2,3}$

Actinic keratosis results from the adverse effects of UV radiation on keratinocyte DNA, reducing the skin's immunity and thus allowing the lesion development. The mutations p16 (INK4a; 9p21), p14 (ARF), p15 (INK4b), and p53 are closely associated with increased risk of progression to squamous cell carcinoma (SCC). ${ }^{4}$

Clinically, the lesions grow slowly and are characterized by erythematous papules, pigmented or not, that can be covered with yellowish or brownish scales, with little or no infiltration, measur-

$\overline{\text { Received } 09 \text { February } 2017 .}$

Accepted 22 May 2017.

* Work conducted at Hospital do Servidor Público Estadual de São Paulo, São Paulo (SP), Brazil.

Financial support: None.

Conflict of interest: The medication was provided to patients free of cost by LEO laboratory.

Dermatology Service, Hospital do Servidor Público Estadual de São Paulo, São Paulo (SP), Brazil.

Dermatology Division, Hospital das Clínicas da Faculdade de Medicina de Ribeirão Preto da Universidade de São Paulo, São Paulo (SP), Brazil.

Dermatology Service, Hospital Alemão Oswaldo Cruz, São Paulo (SP), Brazil.

MAILING ADDRESS:

Maria Isabel Ramos Saraiva

E-mail: isabelsaraivadermato@gmail.com

(C)2018 by Anais Brasileiros de Dermatologia 
ing less than $1 \mathrm{~cm}$ in diameter. The adjacent skin can show evidence of chronic sun damage with telangiectasias, elastosis, wrinkles, poikiloderma, and dyschromia. ${ }^{2}$

AKs can regress spontaneously, remain stable, or transform into invasive SCC. Currently, AKs are considered intraepithelial neoplasms of keratinocytes, with a cumulative risk (5-20\%) of evolving to invasive neoplasm within 10-25 years, with the number of lesions maximizing this occurrence. An estimated $27-82 \%$ of cutaneous SCCs evolve from AK, and $82-97 \%$ of SCCs present contiguous AKs. ${ }^{2}$ Various methods can be used to treat atypical keratinocytes in order to reduce the risk of invasive SCC. ${ }^{1}$

The concept of "field cancerization" suggests that apparently normal skin adjacent to the AK area already presents genetic alterations associated with carcinogenesis. Patients with AK typically have multiple lesions, reflecting local sun damage. ${ }^{5}$ Based on this concept, the management is divided into therapies targeted to the lesion and the field.

The therapies targeted to the lesion include cryotherapy, trichloroacetic acid (TAA), curettage with electrocoagulation, shaving, and excision with suturing. Treatment strategies for the field cancerization include resurfacing techniques (dermabrasion, chemical peeling, and laser), and application of topical medications such as imiquimod $3.75-5 \%$, 5-fluorouracil $0.5-5 \%$, diclofenac sodium 3\%, photodynamic therapy with 5-aminolevulinic acid, and more recently, ingenol mebutate (IM) ${ }^{4-6}$

IM is a macrocyclic diterpene ester, obtained from the natural extract of the plant Euphorbia peplus. IM is the drug most recently approved by the U.S. Food and Drug Administration (FDA) for the treatment of non-hyperkeratotic and non-hypertrophic AKs in

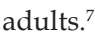

This new drug presents two mechanisms of action: rapid induction of cell death in the treated area a few hours after application and an inflammatory response in a few days after application, capable of eliminating the residual cells. ${ }^{8}$

The keratinocyte's death appears to be related to necrosis through mitochondrial edema and chemoablation with rupture of the plasma membrane. The inflammatory response is caused by both the cell necrosis and activation of protein kinase C, capable of stimulating the release of proinflammatory cytokines, expression of endothelial adhesion molecules, and production of tumor-specific antibodies and leading to cytotoxicity mediated by antibody-dependent neutrophils. ${ }^{8}$

The drug in gel form is available in two presentations, one at a concentration of $150 \mu \mathrm{g} / \mathrm{g}$ for application on the target area of the face or scalp once a day for three consecutive days, and the other at $500 \mathrm{\mu g} / \mathrm{g}$ for use on the trunk or extremities for two consecutive days. The tube's content should be applied on an affected area measuring a maximum of $25 \mathrm{~cm}^{2}$.

The most common adverse reactions include erythema desquamation, and formation of crusts. These reactions are most intense from the third to eighth day from initiation of treatment, with spontaneous improvement within two to four weeks. Edema, formation of pustules, erosion, and ulceration can also occur. There are no reports of scarring. ${ }^{8,9}$

Anderson et al. demonstrated statistically significant im- provement in patients treated with IM when compared to controls (placebo) 57 days after initiating treatment. ${ }^{9}$

There is growing interest in combined therapies for AKs, especially with the advent of new treatment options. However, the final choice of treatment should assess not only efficacy, but also the adverse reactions, aesthetic results, access, cost, and patient's choice. $^{4}$

This study aims to assess the clearance of lesions and the adverse events in the treatment of actinic keratoses with ingenol mebutate used on the face/scalp and limbs.

\section{METHOD}

This was a longitudinal, prospective, non-randomized, non-placebo-controlled, interventional, open, single-center study approved by the Institutional Review Board indicated by the Brazil Platform under case review number 42933914.9.0000.5463. The study only enrolled patients that agreed to participate by signing the free and informed consent form and authorizing use of the photographs of the treated areas.

Complete treatment was provided free of cost to patients by the product's manufacturer. Conflict of interest: provision of the medication by the manufacturer. Eligibility criteria included age over 18 years, willingness to collaborate, and presence of 4 to 8 typical, non-hypertrophic, contiguous actinic keratosis lesions on an area of up to $25 \mathrm{~cm}^{2}$ on the face, scalp, or upper limbs, not previously or currently treated. Subjects neither had a known history of allergy or sensitivity to any component of the formula, nor were using immunosuppressants.

Patients were divided into 2 groups. The first consisted of patients with lesions on the face or scalp and the second, patients with lesions on the limbs. Patients in the first group received IM $150 \mathrm{mcg} / \mathrm{g}$ for three consecutive days, while the second group received IM $500 \mathrm{mcg} / \mathrm{g}$ for two consecutive days. The product was applied by physicians on D1, and the remaining doses were applied by the patients themselves at home.

On each treatment day, the tube's content was applied on an area measuring $25 \mathrm{~cm}^{2}$, spread homogeneously, and left to dry for 15 minutes. Patients were instructed to wash their hands immediately after the application, not to touch the treated area for six hours or expose the area to sun, to store the tubes under refrigeration (2 to $8^{\circ} \mathrm{C}$ ) until their use, and to discard them after opening and use.

Patients were assessed 7 days (D7) after initiating application of the medication, and photographic records were performed, along with analysis of adverse events (pain, local infection, edema, ulcer, pruritis, erythema, desquamation, and paresthesia).

Patients also returned at 57 days (D57) following initiation of treatment for the final evaluation and a new photographic record. At this final follow-up, the treated area was analyzed for clinical clearance of lesions based on counting the residual AKs. We assessed the mean percentage reduction in the lesions, with treatment response classified as follows: complete clearance (100\% improvement in the AKs), i.e., no residual AK; partial clearance $(>75 \%$ improvement of AKs), or a decrease of more than $75 \%$ in the number of AKs; without clearance (improvement $<75 \%$ ), or resolution of less than $75 \%$ in the initial number of AKs. Persistent adverse events and 
sequelae secondary to inflammation from the medication were also assessed.

Exclusion criteria were: patients with lesions suspected of basal cell carcinoma or squamous cell carcinoma within $15 \mathrm{~cm}$ from the area targeted for treatment; other skin conditions that interfered in the evaluation of the treatment evolution; or unwillingness to participate. The study also excluded patients that failed to attend all the scheduled appointments or to complete the treatment. The descriptive analysis only considered patients that concluded the protocol.

\section{RESULTS}

Thirty-one patients were initially selected to participate in the study, of whom 15 patients were assigned to application on the face or scalp and 16 for application on the forearm. Three patients were eventually excluded from the study for not appearing on all the scheduled days, and one for not using the medication correctly.

\section{Group 1: Face and scalp}

This group consisted of 13 patients from 65 to 86 years of age (mean 74.5 years), including $53.8 \%$ men and $46.2 \%$ women. According to the Fitzpatrick classification, $38.5 \%$ were phototype I; $53.8 \%$, phototype II; and $7.7 \%$ phototype III. The number of AK lesions on the target area ranged from 4 to 7 , with a mean of 5.1 lesions. Complete clearance of the lesions occurred in $53.8 \%$ of the treated patients; $15.4 \%$ showed partial clearance; $30.8 \%$ had improvement less than or equal to $50 \%$. Thus, when effective improvement was defined as clearance of more than $75 \%$ of the lesions, $69.2 \%$ of the treated patients showed a good response (Figure 1). There was a mean reduction of $75.8 \%$ when compared to the baseline values in the number of AKs in the treated patients. The most common adverse effects observed at D7 after application of IM were erythema, edema, desquamation, pruritis, and erosion on the application site (Figure 2). The majority of the adverse effects were transient, with spontaneous resolution observed at D57, with persistent erythema and desquamation in only $15 \%$ of the patients, as shown in table 1.

\section{Group 2: Forearm}

The 14 patients allocated to this group ranged in age from 61 to 88 years, with a mean of 73.2 years. Of these, $64.3 \%$ were men and $35.7 \%$ women. As to Fitzpatrick classification, $28.6 \%$ were phototype I; $50 \%$ phototype II; and $21.4 \%$ phototype III. The number of AK lesions in the target area varied from four to eight, with a mean of 6.5 lesions. There was complete clearance of the lesions in $42.8 \%$ of the patients in this group; $35.7 \%$ showed partial clearance; $21.5 \%$ showed improvement between 25 and $50 \%$. Thus, $78.5 \%$ of the patients showed good response to treatment, with clearance of more than $75 \%$ of the lesions (Figures 3 and 4). There was a mean reduction of $87.9 \%$ compared to baseline in the number of AKs in the treated patients. The most common adverse effects observed at D7 after application of ingenol mebutate were erythema, edema, desquamation, and pruritis at the application site, the majority of which were transient, with spontaneous resolution observed at D57, with persistent erythema in $28.6 \%$ of the patients and edema in $7.1 \%$, as shown in table 2 .
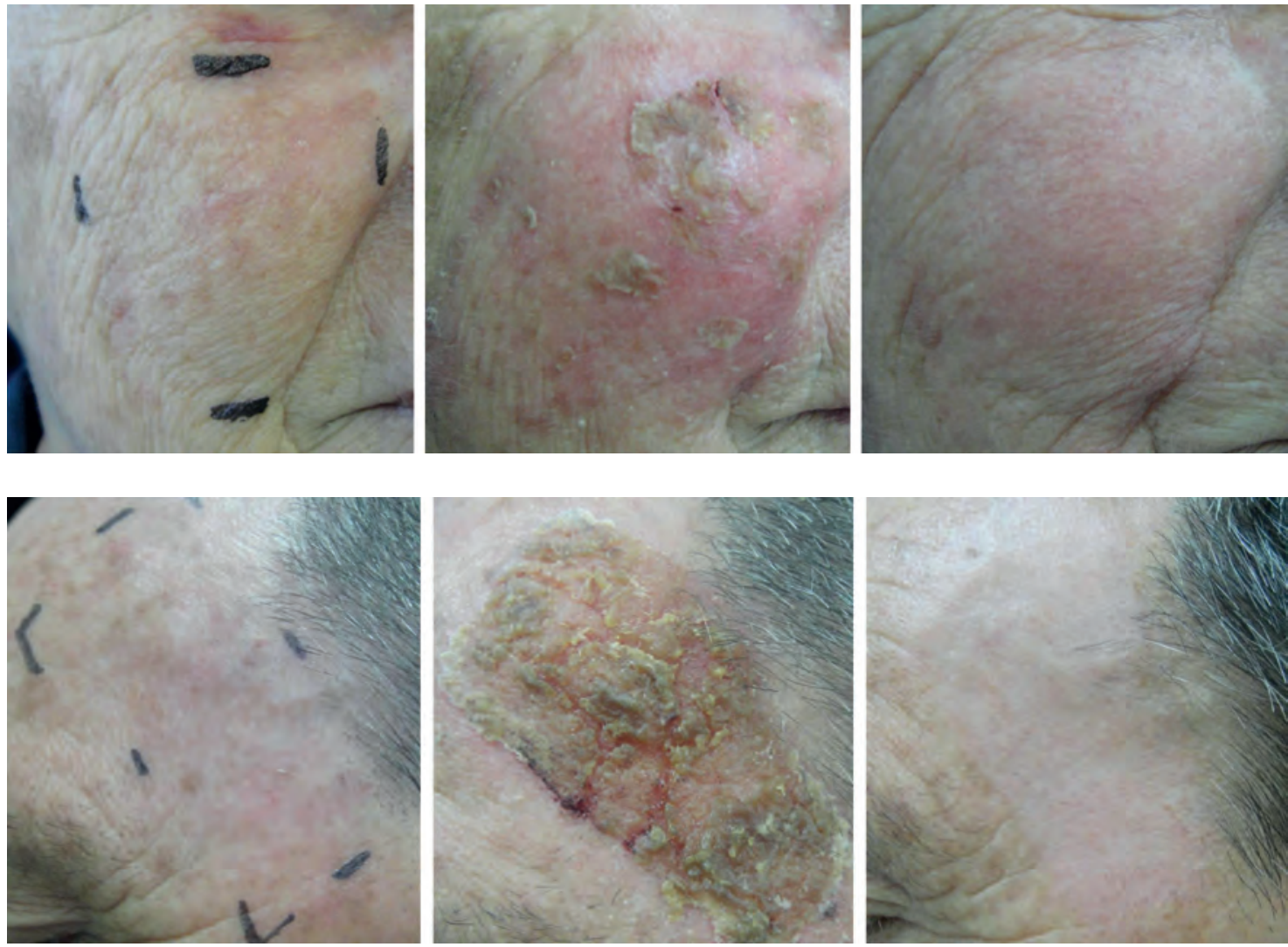

Figure 1: D1, D7, and D57 in right malar region treated for three days with IM $0.015 \%$. On D1 there were four AK lesions, while on D57 there were no lesions. On D7, intense erythema, edema, desquamation, and crusting

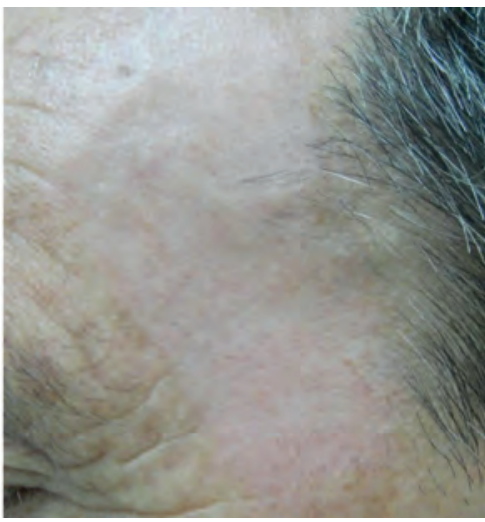

FiguRE 2: D1, D7, and D57 in left temporal region treated for three days with IM $0.015 \%$. On D1 there were four AK lesions, while on D57 there were no lesions. On D7, mild erythema, desquamation, and crusting 


\section{DISCUSSION}

Topical treatment of field cancerization for AK applied by the patient should be effective, safe, and user-friendly in order to allow completing the treatment. ${ }^{8}$

In this study, treatment with IM gel $0.015 \%$ or $0.05 \%$ applied on the face/scalp or forearm, respectively, was found to be well-tolerated, and the adverse effects were considered mild and mostly transient. Anderson et al., reported similar findings, with partial clearance in patients treated with IM in non-facial areas varying from 56 to $75.4 \%$ compared to $21.7 \%$ with the vehicle. The complete response rate was also significantly higher in patients treated with IM, i.e., from 40 to $54.4 \%$, compared to $1.7 \%$ improvement in the group treated with the vehicle. ${ }^{9}$ Lebwohl et al. demonstrated the superiority of IM in lesions on the trunk and extremities, with $49.1 \%$ partial improvement compared to $6.9 \%$ in the placebo group. Complete response was seen in $34.1 \%$ in the IM group, compared to $4.7 \%$ in the placebo group. Median reduction in the number of lesions was $75 \%$ in the group treated with IM, compared to $0 \%$ in the placebo group. Lesions on the face and scalp showed complete improvement in $42.2 \%$ at D57 in patients treated with IM, compared to $3.7 \%$ in the placebo group. The treated group showed partial improvement in $63.9 \%$ of the patients, compared to $7.4 \%$ in the placebo group. Median reduction in the number of lesions was $83 \%$ in the treated group, compared to zero response in the placebo group. ${ }^{8}$

There are numerous topical options currently available for the treatment of field cancerization in AK, with various dosages, treatment times, efficacy, and adverse event profiles. Stockfleter et al., in a systematic review on the use of topical treatments for $\mathrm{AK}$, performed a qualitative comparison showing complete clinical response with 5-fluorouracil (55.5\%), followed by IM (42.2\%), and imiquimod $3.75 \%$ (25-35.6\%). ${ }^{10}$ Studies that used 5-fluorouracil treated hyperkeratotic lesions, which are more aggressive, with greater potential for malignant transformation, while studies with IM and imiquimod did not. ${ }^{10}$

Gupta et al. reviewed various therapeutic interventions for AK, aimed at treatment of field cancerization. The different therapies that were analyzed (diclofenac 3\%, 5-fluorouracil 0.5\%, imiqui$\bmod 5 \%$, and IM at $0.015 \%$ and $0.05 \%$ ) showed similar efficacy but were associated with different cosmetic outcomes and adverse effects, with the following dropout rates: 144 of 1,000 participants in the group treated with diclofenac sodium in hyaluronic acid $2.5 \%$; 40 of 1,000 participants using only hyaluronic acid $2.5 \%$; and 56 of 1,000 using imiquimod $5 \%$, compared to 21 of 1,000 patients that received placebo. The study did not report the dropout rate for patients that used IM. The authors suggested that more studies were needed to compare the various treatment modalities. ${ }^{11}$ Sotiriou et al. used photodynamic therapy with aminolevulinic acid for treatment of field cancerization and observed a $65.32 \%$ response rate in six months of follow-up for two treatment sessions. ${ }^{12}$ Samorano et al. found that local reactions with IM were less lasting when compared to 5- fluorouracil, but that both were safe and well-tolerated. ${ }^{13}$

In our study, all the patients had adverse effects at D7, which were expected for the treatment. There was good tolerability, with $33.3 \%$ presenting prolonged adverse effects (erythema $25.9 \%$, desquamation $7.4 \%$, scaling $7.4 \%$, pruritis $7.4 \%$, periorbital edema $3.7 \%$, and hypochromia $3.7 \%$ ), none of which were considered serious. The persistence of these effects had already been observed in a previous study, showing alterations in pigmentation in all the study groups, besides minimal scarring. ${ }^{8}$ Patients treated with IM that presented higher grade inflammation resulting from the medication ap-

TABLE 1: Adverse effects observed on the face at days 7 and 57

\begin{tabular}{ll} 
Adverse effect on face & D7 D57 \\
\hline Infection & $15.4 \%$ \\
Headache & $15.4 \%$ \\
Periorbital edema & $23.1 \% 7.7 \%$ \\
Erosion & $46.2 \%$ \\
Vesicles & $38.5 \%$ \\
Local edema & $53.8 \%$ \\
Desquamation & $61.5 \% 7.7 \%$ \\
Scaling & $53.8 \% 15.4 \%$ \\
Erythema & $69.2 \% 15.4 \%$ \\
Pain & $38.5 \%$ \\
Pruritis & $84.6 \% 7.7 \%$ \\
Paresthesia & $7.7 \%$ \\
Ulcer & $7.7 \%$ \\
Hypochromia & $7.7 \%$ \\
\hline
\end{tabular}
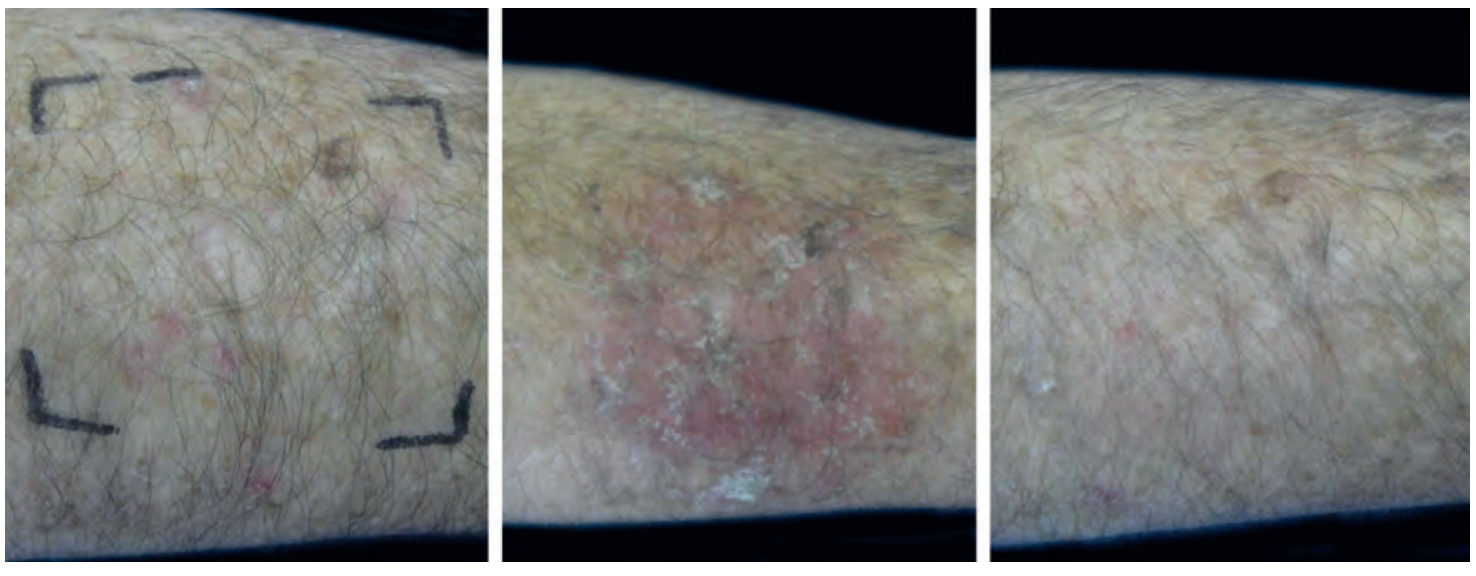

Figure 3: D1, D7, and D57 on forearm treated for two days with IM $0.05 \%$. On D1 there were six AK lesions, while on D57 there was one lesion. On D7, intense erythema, desquamation, and crusting 

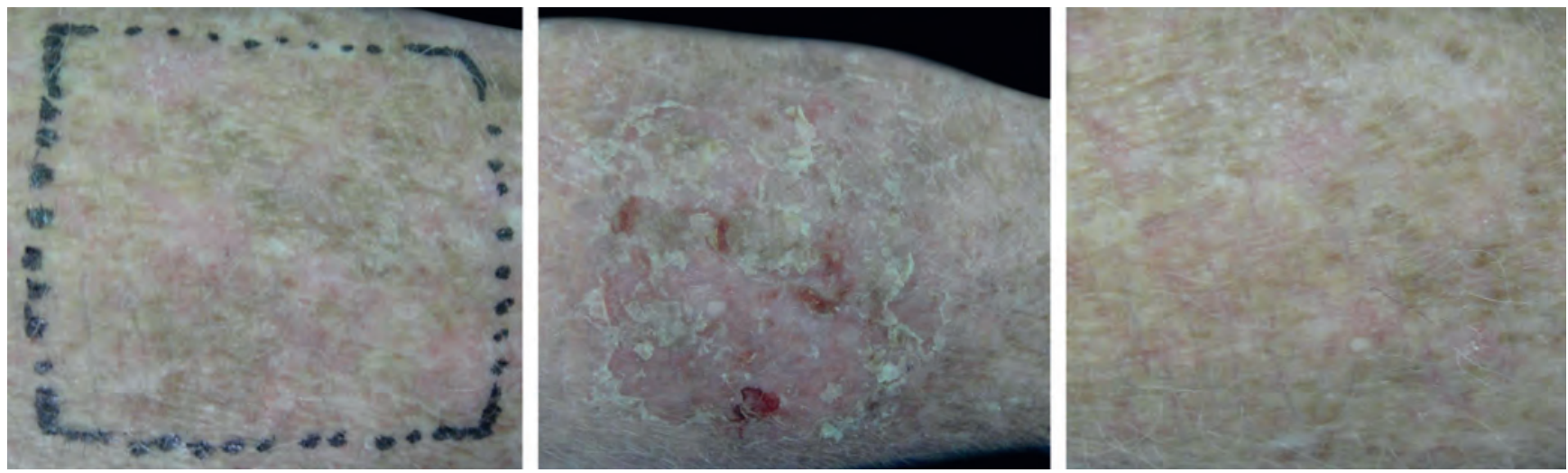

Figure 4: D1, D7, and D57 on forearm treated for two days with IM 0.05\%. On D1 there were six AK lesions, while on D57 there was one lesion. On D7, moderate erythema, desquamation, crusting

\begin{tabular}{ll}
\multicolumn{2}{c}{ TABLE 2: Adverse effects observed on the forearm at } \\
days 7 and 57
\end{tabular}

pear to have evolved with more significant clinical improvement of the treated area, based on aesthetic and therapeutic improvement, but more studies are necessary.

As for dosage, we observed high treatment adherence, since $90 \%$ of patients completed treatment, appearing for follow-up at both D7 and D57, which had been reported previously by Matin and Swanson. ${ }^{14}$ The dosing schedule can be advantageous when compared to other drugs, since it requires shorter treatment times, and the adverse effects only began after completing the prescribed treatment regimen. It is thus recommended to apply the product in the physician's office or clinic in order to avoid inadequate use of the medication. The main advantage of treatment with ingenol mebutate is that similar degrees of efficacy can be achieved with only two or three days of treatment. ${ }^{8}$

The study had the following limitations: low number of patients, lack of blinding, lack of placebo control, no direct comparison with other medications, and lack of follow-up at one year. Neither did the study assess the reasons for patients' failure to appear on the scheduled days, and no biopsies were performed to prove the diagnosis prior to treatment, nor after treatment to prove clinical improvement. Since the treatment was performed on exposed areas like the face, we opted to perform diagnostic and follow-up exclusively according to clinical and dermoscopic criteria. Since the study was not a randomized clinical trial, it did not assess efficacy and safety, but it does corroborate some findings already published in the world literature. ${ }^{8}$

\section{CONCLUSION}

Patients that used ingenol mebutate showed good clearance of the lesions and foreseeable adverse events. The short treatment period appears to have facilitated patients' adherence. We thus conclude that IM can be an option in the therapeutic armamentarium for AK. $\square$

\section{ACKNOWLEDGEMENT}

The authors wish to thank Dr. José Alexandre de Souza Sittart for his encouragement and support for conducting this study. 


\section{REFERENCES}

1. Rigel DS, Stein Gold LF. The importance of early diagnosis and treatment of actinic keratosis. J Am Acad Dermatol. 2013;68:S20-7.

2. Schmitt JV, Miot HA. Actinic keratosis: a clinical and epidemiological revision. An Bras Dermatol. 2012;87:425-34.

3. de Berker D, McGregor JM, Hughes BR; British Association of Dermatologists Therapy Guidelines and Audit Subcommittee. Guidelines for the management of actinic keratoses. Br J Dermatol. 2007;156:222-30.

4. Dodds A, Chia A, Shumack S. Actinic keratosis: rationale and management. Dermatol Ther (Heidelb). 2014;4:11-31.

5. Costa C, Scalvenzi M, Ayala F, Fabbrocini G, Monfrecola G. How to treat actinic keratosis? An update. J Dermatol Case Rep. 2015;9:29-35.

6. Torezan L, Chaves Y, Niwa A, Sanches JA Jr, Festa-Neto C, Szeimies RM. A pilot split-face study comparing conventional methyl aminolevulinate-photodynamic therapy (PDT) with microneedling-assisted PDT on actinically damaged skin. Dermatol Surg. 2013;39:1197-201.

7. Micali G. Nasca MR, De Pasquale R. Erythroplasia of Queyrat treated with imiquimod 5\% cream. J Am Acad Dermatol. 2006;55:901-3.

8. Lebwohl M, Swanson N, Anderson LL, Melgaard A, Xu Z, Berman B. Ingenol mebutate gel for actinic keratosis. N Engl J Med. 2012;366:1010-9.
9. Anderson L, Schmieder GJ, Werschler WP, Tschen EH, Ling MR, Stough DB, et al. Randomized, double-blind, double-dummy, vehicle-controlled study of ingeno mebutate gel $0.025 \%$ and $0.05 \%$ for actinic keratosis. J Am Acad Dermatol. 2009;60:934-43.

10. Stockfleth E, Sibbring GC, Alarcon I. New Topical Treatment Options for Actinic Keratosis: A Systematic Review. Acta Derm Venereol. 2016;96:17-22.

11. Gupta AK, Paquet M, Villanueva E, Brintnell W. Interventions for actinic keratoses. Cochrane Database Syst Rev. 2012;12:CD004415.

12. Sotiriou E, Apalla Z, Maliamani F, Zaparas N, Panagiotidou D, loannides D. Intraindividual, right-left comparison of topical 5-aminolevulinic acid photodynamic therapy vs. $5 \%$ imiquimod cream for actinic keratoses on the upper extremities. $J$ Eur Acad Dermatol Venereol. 2009;23:1061-5.

13. Samorano LP, Torezan LA, Sanches JA.Evaluation of the tolerability and safety of a $0.015 \%$ ingenol mebutate gel compared to $5 \% 5$-fluorouracil cream for the treatment of facial actinic keratosis: a prospective randomized trial. J Eur Acad Dermatol Venereol. 2015;29:1822-7.

14. Martin G, Swanson N. Clinical findings using ingenol mebutate gel to treat actinic keratoses. J Am Acad Dermatol. 2013:68:S39-48.

\section{AUTHORS CONTRIBUTION}

Maria Isabel Ramos Saraiva

(iD) ORCID 0000-0002-5043-489X

Statistical analysis; Approval of the final version of the manuscript; Elaboration and writing of the manuscript; Obtaining, analyzing and interpreting the data; Intellectual participation in propaedeutic and/or therapeutic conduct of the cases studied; Critical participation in propaedeutic and/or therapeutic conduct
review of the literature; Critical review of the manuscript
Larissa Karine Leite Portocarrero
iD ORCID
0000-0003-3357-1013

Statistical analysis; Approval of the final version of the manuscript; Elaboration and writing of the manuscript; Obtaining, analyzing and interpreting the data; Intellectual participation in propaedeutic and/or therapeutic conduct of the cases studied; Critical review of the literature; Critical review of the manuscript

Marcella Amaral Horta Barbosa Vieira

iD ORCID 0000-0003-2892-9922

Statistical analysis; Approval of the final version of the manuscript; Elaboration and writing of the manuscript; Obtaining, analyzing and interpreting the data; Intellectual participation in propaedeutic and/or therapeutic conduct of the cases studied; Critical review of pation in propaedeutic and/or therapeutic con
the literature; Critical review of the manuscript

\section{Bethania Cabral Cavalli Swiczar}

Approval of the final version of the manuscript; Conception and planning of the study; Effective participation in research orientation; Intellectual participation in propaedeutic and/or therapeutic conduct of the cases studied; Critical review of the manuscript

Andrezza Telles Westin

$$
\text { (iD) ORCID 0000-0003-4256-208X }
$$

Approval of the final version of the manuscript; Conception and planning of the study; Obtaining, analyzing and interpreting the data; Effective participation in research orientation; Intellectual participation in propaedeutic and/or therapeutic conduct of the cases studied; Critical review of the manuscript

How to cite this article: Saraiva MIR, Portocarrero LKL, Vieira MAHB, Swiczar BCC, Westin AT. Ingenol mebutate in the treatment of actinic keratoses: clearance rate and adverse effects. An Bras Dermatol. 2018;93(4):529-34 